\title{
Classificação de risco de família: uma ferramenta de avaliação para equipes da Estratégia Saúde da Família
}

Danielle Freitas Alvim de Castro, M.Sc.*, Alder Mourão de Sousa, M.Sc.**, Lislaine Aparecida Fracolli***

*Enfermeira, Doutoranda em Ciências pela Escola de Enfermagem da USP, Bolsista CAPES, **Enfermeiro, Escola de Enfermagem da USP, ***Enfermeira, Profa. do Departamento de Enfermagem em Saúde Coletiva da Escola de Enfermagem da USP

\section{Resumo}

Uma das fragilidades da Estratégia Saúde da Família (ESF) refere-se ao fato de que a prática profissional ainda está pautada no atendimento individualizado ao membro familiar doente. Devido a isso se faz necessário instrumentalizar os profissionais das equipes com vistas à inclusão da abordagem familiar nas açôes de saúde no território onde atuam. O objetivo desta pesquisa foi validar um instrumento de classificação de famílias em uma UBS com a ESF. Trata-se de uma pesquisa quantitativa com desenho metodológico do tipo estudo de caso. Foram sujeitos da pesquisa 230 famílias. Os resultados mostraram que 58,7\% das famílias encontravam-se na faixa de risco moderado, 36,52\% na faixa de maior risco e 4,78\% na faixa de baixo risco. Conclui-se que esse instrumento é uma potente ferramenta para auxiliar as equipes de ESF na realização do diagnóstico de saúde local, como instrumento auxiliar para o planejamento e avaliação das açóes.

Palavras-chave: Programa Saúde da Família, família, avaliação em saúde.

\section{Abstract}

\section{Family risk classification: an assessment tool for Family Health Strategy teams}

One of the Family Health Strategy (FHS) weaknesses refers to the fact that they are still using individualized care to a sick family member. Consequently, health professional teams should have conditions to include family approach in health care in the territory where they operate. The objective of this research was to validate a classification tool in a Health Basic Unit with FHS. This is a quantitative research with case study methodology. We interviewed 230 families. The results showed that $58.7 \%$ of families were in the moderate risk category, $36.52 \%$ in higher risk and $4.78 \%$ lower risk. It is concluded that this instrument is a powerful tool to help teams of Family Health to carry out the community health diagnosis, as an aid for planning and evaluating actions.

Key-words: Family Health Program, family, health evaluation.

\section{Resumen}




\section{Clasificación de riesgo familiar: un instrumento de evaluación para los equipos de la Estrategia Salud de la Familia}

Una de las fragilidades de la Estrategia Salud Familiar (ESF) se refiere al hecho de que la práctica profesional todavía está centrada en la atención individualizada al miembro familiar enfermo. Debido a esto, es necesario dotar a los equipos profesionales de condiciones con el fin de incluir el enfoque de familia en el cuidado de la salud en el territorio donde operan. El objetivo de esta investigación fue validar una herramienta de clasificación de riego familiar en una Unidad Básica de Salud con ESF. Se trata de una investigación cuantitativa con un diseño metodológico de estudio de caso. Se entrevistaron a 230 familias. Los resultados mostraron que el 58,7\% de las familias se encontraban en la categoría de riesgo moderado, 36,52\% de alto riesgo y $4,78 \%$ de leve riesgo. Se concluye que este instrumento es una potente herramienta para ayudar a los equipos de Salud de la Familia en el diagnóstico local de salud, como instrumento auxiliar para la planificación y evaluación de las acciones.

Palabras-clave: Programa de Salud Familiar, familia, evaluación en salud.

\section{Introdução}

A Estratégia Saúde da Família (ESF) foi planejada como uma alternativa para a consolidação da Atenção Básica no contexto do SUS. Ela é considerada um modelo de Atenção Primária à Saúde focada na unidade familiar e construída operacionalmente na esfera comunitária $[1,2]$. Preconiza a organização do trabalho a partir do atendimento de famílias de uma determinada área adscrita, desenvolvendo ações de promoção da saúde, prevenção, recuperação, reabilitação de doenças e agravos mais frequentes, e na manutenção da saúde desta comunidade [3].

Ermel e Fracolli destacam que a grande fragilidade dessa estratégia refere-se ao fato da prática profissional ainda estar pautada no atendimento individualizado ao membro familiar doente [4]. Os manuais e protocolos de atendimento para os profissionais que atuam nessa estratégia não orientam a estruturação do cuidado familiar, não fazem referência à diversidade de composiçóes, tamanho, dinâmica, papéis e funçóes, necessidades e estratégias de sobrevivência das famílias na atualidade. Também não discutem meios que ajudem a abordagem, condução e sistematização do atendimento familiar no cotidiano das equipes [5].

Embora a ESF se constitua em uma importante e inovadora maneira de se fazer frente à transição epidemiológica pela qual passa a população brasileira, onde as doenças e agravos não transmissíveis assumem um protagonismo frente às doenças transmissíveis, faz-se necessário instrumentalizar os profissionais das equipes de saúde com vistas à inclusão da abordagem familiar no planejamento das açôes de saúde no território onde atuam.

Para se desenvolver a abordagem de família no cotidiano dos profissionais que atuam na ESF pode-se lançar mão de tecnologias específicas como o genograma e o ecomapa, instrumentos que possibilitam uma rápida visualização dos integrantes $\mathrm{da}$ família, das relaçôes de parentesco entre eles e das relaçôes deles com a comunidade onde vivem, facilitam a recuperação de informações, oportunizando melhores planejamentos e discussão de casos. Estes instrumentos podem ser acrescidos de outros dados que a equipe considerar importantes, como idade, patologia de base etc., e ainda do grau e qualidade das relaçôes mantidas entre os membros [6].

Outra potente tecnologia disponível é a avaliação e intervenção familiar proposta por Wright e Leahey, enfermeiras da Universidade de Calgary no Canadá, que propóem um modelo para avaliar e intervir (MCAF e MMCIF) junto às famílias. Esse instrumento baseia-se em uma técnica de entrevista de 15 minutos, levando em consideração a limitação de tempo e o grande volume de trabalho das equipes de saúde [7].

Um passo em direção a uma abordagem mais coletiva para o modelo de atenção à saúde da família é realizar a classificação de risco das famílias adscritas, objetivando organizar e priorizar o trabalho levando em consideração a realidade e vulnerabilidade experienciada por cada família [8].

O Instrumento de Classificação de Risco, adaptado por Craco e proposto por Vieira apud Craco, foi utilizado como estratégia para o manejo de iniquidades, com a finalidade de classificar as famílias de acordo com suas reais necessidades. Além disso, permite distinção das famílias de acordo com as morbidades encontradas no núcleo familiar, além de utilizar nessa classificação alguns itens socioeconômicos, classificando-as em famílias de risco maior 
(cor vermelha), risco moderado (cor amarela) e baixo risco (cor verde) [8].

Na Família Vermelha (ou de maior risco) encontramos os seguintes itens de risco familiar:

- Acidente vascular cerebral

- Baixas condiçóes econômicas

- Bebês < 6 meses

- Colesterol alto

- Criança sem escola

- Criança $<1$ ano sem acompanhamento

- Diabetes com complicaçóes

- Doenças graves descontroladas ou descompensadas

- Gestação de alto risco

- Tabagismo grave

- Tabagismo com infecção respiratória

- Idoso solitário, acamado

- Infarto Agudo do Miocárdio

- Obesidade

- Pessoas com câncer

- Pobreza extrema

- Problemas mentais

- Trombose

- Violência doméstica, abuso sexual

- Problemas cardíacos

- Etilismo grave

Na Família Amarela (ou de risco moderado) encontramos os seguintes itens de risco familiar:

- Deficiência física

- Diabetes controlada

- Depressão leve

- Doenças graves controladas ou leves descontroladas

- Epilepsia

- Problemas gástricos

- Gestante em acompanhamento

- Hipertensão controlada

- HIV com acompanhamento

- $<1$ ano e adolescentes

- Problemas circulatórios

- Problemas na coluna

- Problemas respiratórios

- Problemas neurológicos

- Problemas ginecológicos

- Problemas hematológicos

- Problemas endócrinos

- Osteoporose

- Alergia

- Elitismo

- Tabagismo
- Problemas articulares

- Patologia prostática

- Reumatismo

Ao se deparar com itens do tipo "gestação em acompanhamento" podemos pensar que isso é uma situação positiva e não de risco. $\mathrm{O}$ risco embutido nesse instrumento são exatamente as situaçôes que deixam a família mais vulnerável, no caso a própria gestação.

Na Família Verde (ou de baixo risco) encontramos os itens:

- Aparentemente náo possui problemas

- Visita domiciliar mensal

Craco ao adaptar este instrumento de classificação de risco de famílias, aplicou-o na região Ribeirão Preto/SP. Para esta autora sua utilização possibilita às equipes de SF organizarem melhor a assistência às famílias no sentido de possibilitar a adoção de medidas que priorizem ações para famílias em maior risco [8].

A possibilidade de testar a utilização deste instrumento e de analisar suas potencialidades e limites para a organização do trabalho com as famílias na ESF foi o que motivou a presente pesquisa.

\section{Objetivos}

Classificar as famílias adscritas às equipes da ESF de uma UBS na cidade de São Paulo, segundo o instrumento de classificaçáo de risco adaptado por Craco [8].

Analisar as potencialidades e limites dessa classificação para a organização de uma assistência com base na família.

\section{Material e métodos}

Tratou-se de uma pesquisa quantitativa, com desenho de estudo de caso, cuja avaliação da Classificação de Risco foi realizada utilizando o Instrumento de Classificação de Risco de Famílias.

O cenário de estudo foi a Unidade Básica de Saúde do Jardim Boa Vista. Essa UBS pertence à administração regional do Butantá, no distrito Raposo Tavares, localizado na periferia da região oeste da cidade de São Paulo. Possui seis equipes de composição mínima da Estratégia Saúde da Família implantadas desde maio de 2002. 
Participaram da pesquisa as famílias adscritas nas áreas de abrangência das equipes Azul e Verde, num total de 230 famílias, sendo 130 da equipe Azul e 100 da equipe Verde. Foram considerados como sujeitos os moradores que possuíssem mais de 18 anos e que concordassem em participar da pesquisa, sendo que só poderiam participar um morador por família.

$\mathrm{Na}$ área da equipe Azul concentram-se famílias de baixo poder aquisitivo, chegando a ter 15 casas em um único terreno, algumas construídas com material reaproveitável. Na área Verde concentram-se famílias de classe média e destaca-se um condomínio residencial com boa infraestrutura.

Anterior à coleta de dados, a pesquisa teve sua aprovação no Comitê de Ética em Pesquisa da Secretaria Municipal de Saúde da Paulo $\left(\mathrm{n}^{\circ}\right.$ 0124.0.162.000-07) e foi realizada uma reunião com as equipes responsáveis pelas áreas pesquisadas. Com a presença dos ACSs de cada área foram visitadas $8,26 \%$ dos moradores da equipe verde e $15,1 \%$ dos moradores da equipe azul e aplicado o instrumento ao adulto que se dispôs a participar da pesquisa tendo-se seguidos os preceitos éticos de pesquisa com seres humanos.

O instrumento foi aplicado em forma de entrevista, onde o adulto participante era convidado a responder afirmativa ou negativamente sobre a existência dos itens contidos no instrumento. A família com um item no risco maior já era considerada Família Vermelha, mesmo que possuísse mais itens no risco moderado, pois esse único item já a classificava em um grau maior de vulnerabilidade.

Os dados foram analisados através do registro da frequência simples das informaçóes obtidas com o auxílio do software Statistical Package for the Social Sciences (SPSS) versão 14.0.

\section{Resultados e discussão}

De acordo com o Instrumento de Classificação de Risco de Famílias, mais da metade das famílias entrevistadas se encontravam no risco de saúde Amarelo, uma parcela significativa no maior risco de saúde (Vermelho), e uma pequena parcela de famílias no risco de saúde Verde, ou seja, sem risco aparente. O gráfico a seguir apresenta a divisão das famílias participantes em relação à classificação de risco.
Gráfico 1 - Classificação do Risco de Família Geral.

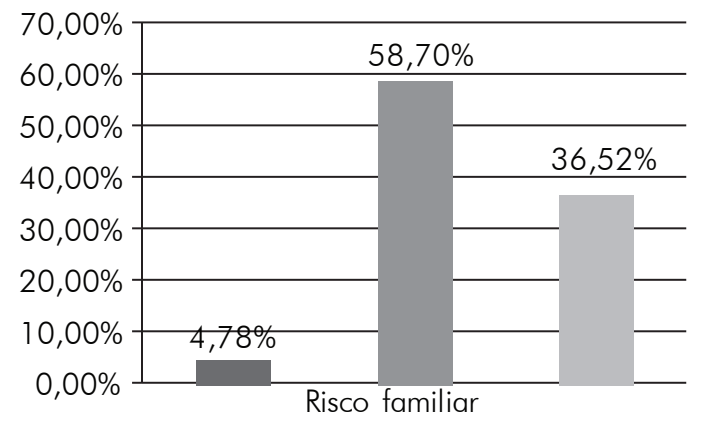

-Família verde $\quad$ Família amarela $\quad$ Família vermelha

Apesar de a área Azul ser considerada uma área de maior vulnerabilidade, nesta pesquisa, constata-se que há uma maior porcentagem de famílias na faixa de maior risco nas famílias da área Verde como mostra os Gráficos 2 e 3.

Gráfico 2 - Classificação de Risco de Família - Equipe Azul.

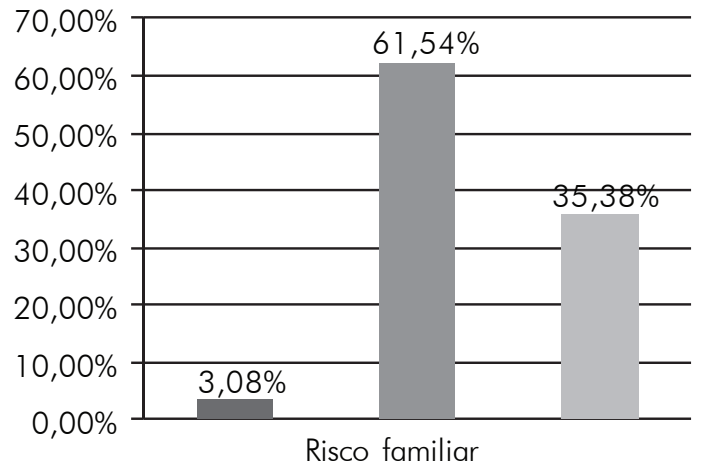

-Família verde Família amarela $\square$ Família vermelha

Gráfico 3 - Classificação de Risco de Família - Equipe Verde.

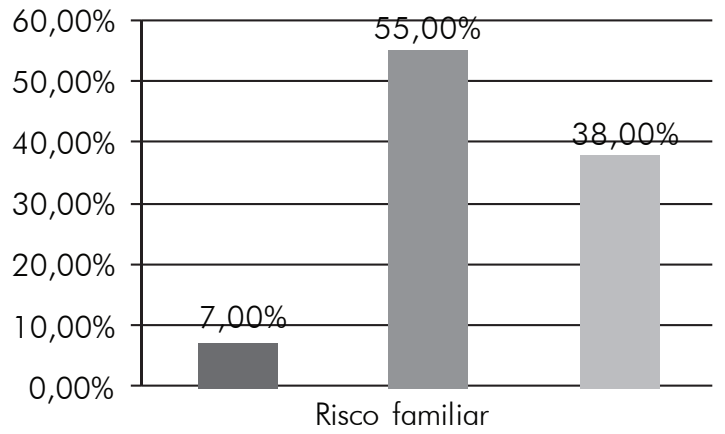

-Família verde Família amarela Família vermelha

Este fenômeno pode ser resultado do próprio processo histórico da área Azul, na qual ocorre uma mobilização dos moradores através de sua Associação para conquista de maior número de vagas nas 
creches e escolas, e maior acesso aos serviços de saúde públicos.

Dentre os entrevistados havia um predomínio de pessoas entre 26 e 45 anos. Em relação ao sexo, o predomínio foi de mulheres. Comparando esses dados nos leva a pensar no fato de apesar das mulheres estarem conquistando seus espaços na sociedade, ainda há um predomínio de mulheres donas de casa, em especial na periferia dos municípios.

Taschner e Bógus verificaram que no município de Sáo Paulo a proporçáo de chefes mulheres declina nitidamente do centro para a periferia. No anel central, onde residem as famílias de renda mais elevada, a proporçáo de chefia feminina atinge $28,51 \%$, porcentagem maior do que no anel periférico, onde se concentram as famílias mais pobres. Não há, portanto, a possibilidade de se estabelecer uma relação direta entre pobreza e chefia feminina [9]. Em contrapartida, Torres et al. referem que os setores com níveis mais altos do indicador de privação tendem a concentrar a população mais frágil, onde há uma proporção maior de mulheres com baixa escolaridade e chefes de família [10].

Em relação à escolaridade dos usuários, nos deparamos com a realidade das classes menos favorecidas do nosso país, pois mais de $40 \%$ da população entrevistada náo possui o ensino fundamental completo, em contrapartida menos de 5\% dos entrevistados possuem formação em nível superior. Taschner e Bógus reafirmam que a associação da escolaridade com a renda é alta no anel central de São Paulo e extremamente baixa no periférico [9].

Ao se proceder a classificação das famílias segundo o instrumento de risco observou-se que $58,7 \%$ das famílias entrevistadas estão na faixa de risco mediano para a saúde. A grande maioria está nesta faixa porque possuem hipertensão e/ou diabetes controlada, gravidez em acompanhamento e crianças menores de um ano.

Torres et al. referem que os setores com níveis mais altos do indicador de privação tendem a concentrar a população mais frágil. Esses setores apresentam renda média mais baixa e índice de escolaridade inferior, maior proporção de famílias muito pobres, maior número médio de habitantes por domicílio, proporção maior de adolescentes. Dessa maneira tornam-se famílias em situaçóes socialmente vulneráveis, pois a menor escolaridade significa uma menor capacidade de emprego novo, além de subemprego nos casos de pessoas empregadas. Mulheres chefes de família e com uma escolaridade mais baixa indicam uma renda familiar menor devido à discrimição na qual as mulheres sofrem no mercado de trabalho, podendo significar também a única fonte de renda familiar. Rendas menores significam condiçôes piores de nutrição e moradia, o que leva a uma probabilidade maior de doenças infecciosas e respiratórias, o que pode gerar um impacto significativo na renda familiar. Além de que um número maior de pessoas por domicílio significa que os recursos comuns são partilhados, o que pode trazer consequências para a nutriçáo, saúde e educação dos membros dessa família [10].

Acreditamos na potência deste instrumento para auxiliar as equipes na realização do diagnóstico de saúde local, como instrumento auxiliar para o planejamento e avaliaçáo das açóes. Ressaltamos que o mesmo não substitui a utilização dos relatórios produzidos pelo Sistema de Informação da Atenção Básica (SIAB) e que estes devem ser utilizados em conjunto com objetivo de agregar mais informaçóes para a tomada de decisão.

As famílias classificadas como Vermelha e Amarela devem receber atenção diferenciada. A equipe de saúde deve discutir criteriosamente os casos em suas reunióes, inclusive com o Núcleo de Apoio à Saúde da Família (NASF), e organizar seu quadro de visitas domiciliárias para que além da visita do Agente Comunitário da Saúde (ACS) estas famílias recebam a visita de outro profissional da equipe no período de um mês, além das consultas médicas e de enfermagem segundo a rotina. A escolha do profissional responsável por realizar a visita deve respeitar a necessidade de cada caso em questáo, podendo variar a cada mês. Retornos dos ACS a uma família Vermelha devem ser planejados de forma que estas famílias sejam acompanhadas, minimamente, com frequência quinzenal.

As famílias classificadas na cor Verde devem receber as visitas de forma rotineira respeitando a organização do trabalho.

A possibilidade de atendimento em grupo deve ser analisada, não somente para diminuir o número de atendimentos individuais, mas de forma a compartilhar com as famílias situaçóes e informaçóes que sejam comuns à realidade local.

Considerando a necessidade de acompanhamento específico e diferenciado para as famílias Vermelhas, o que demanda mais tempo e atenção, uma equipe de saúde que possui uma grande quantidade de famílias nesta condição em uma mesma microárea ou em sua área adscrita deve pleitear junto 
aos seus coordenadores uma redução do número total de famílias cadastradas de modo a respeitar os limites humanos de trabalho dos profissionais e de forma a assegurar tempo e disponibilidade para uma atenção diferenciada às necessidades singulares de cada família.

Os indicadores que constituem o instrumento de Classificação de Risco de Famílias apresentados neste artigo são exemplos de possíveis situaçóes e patologias que podem ser encontradas na realidade local de cada equipe de saúde, podendo ser acrescido de outros indicadores conforme a realidade e a necessidade vivenciada em cada área/microárea adscrita.

\section{Conclusão}

Acreditamos que a utilização desta tecnologia possa ajudar as equipes de Saúde da Família no planejamento do trabalho, na elaboração de cronogramas, priorizando a otimizaçáo dos recursos disponíveis para o alcance dos objetivos de cada equipe.

Cremos que as equipes ao optarem pela utilização deste instrumento podem lançar mão de estratégias diferenciadas da utilizada nesta pesquisa. Podendo ser preenchido por etapas, em diferentes encontros e respondido por diferentes pessoas e/ou em conjunto com a família.

Consideramos que existe uma necessidade premente de otimizar recursos e priorizar açóes no cotidiano do processo de trabalho das equipes de saúde da família e destacamos uma alternativa para este fim.

\section{Agradecimentos}

À FAPESP pelo apoio financeiro, aos ACS das equipes Azul e Verde e os profissionais da UBS Jd. Boa Vista.

\section{Referências}

1. Barrêto ICHC, Andrade LOM, Martins Junior T. A estratégia saúde da família no Brasil e a superação de medicina familiar. Sanare 2002;(1):82-7.

2. Andrade LOM, Goya N, Martins Junior T, Barrêto ICHC. Escola de formaçáo em saúde da família Visconde de Sabóia - Sobral (CE): uma resposta municipal para educação permanente no SUS. Divulg Saúde Debate 2004;(30):15-25.

3. Brasil. Ministério da Saúde. Departamento de Atenção. Coordenação de Saúde da Comunidade. Saúde da Família: uma estratégia para o modelo assistencial. Brasília: Ministério da Saúde; 1997. 36p.

4. Ermel RC, Fracolli LA. O trabalho das enfermeiras no Programa de Saúde da Família em Marília/SP. Rev Esc Enferm USP 2006;40(4):533-9.

5. Rodrigues CRF. Representaçôes sociais de trabalhadores da Saúde da Família sobre família como como unidade do cuidado e controle social, por meio dos Conselhos Gestores, Zona Leste da Cidade de Sáo Paulo [Tese]. São Paulo: Faculdade de Saúde Pública; 2009.

6. Bousso RS, Angelo M. A Enfermagem e o Cuidado na Saúde da Família. In: Brasil, Instituto para o Desenvolvimento da Saúde, Universidade de São Paulo, Ministério da Saúde. Manual de Enfermagem. Brasília: Ministério da Saúde; 2001. p.18-22.

7. Wright LM, Leahey M. Nurses and families: A guide to family assessment and intervention. $5^{\mathrm{a}} \mathrm{ed}$. Philadelphia: F.A. Davis; 2009.

8. Craco PF. A Ação Comunicativa no cuidado à saúde da família: encontros e desencontros entre profissionais de saúde e usuários [Tese]. Ribeirão Preto: Universidade de São Paulo Campus de Ribeirão Preto; 2005.

9. Taschner SP, Bógus LMM. São Paulo, uma metrópole desigual. EURE (Santiago) 2001;27(80):87-120.

10. Torres HG, Marques E, Ferreira MP, Bitar S. Pobreza e espaço: padróes de segregação em São Paulo. Estud Av 2003;17(47):97-128. 